\title{
Basic Geometric Concepts in the Thinking of In-Service and Pre-Service Mathematics Teachers
}

\author{
Alessandro Gambini ${ }^{1, *(D)}$ and István Lénárt ${ }^{2}$ \\ 1 Department of Mathematics "Guido Castelnuovo", Sapienza University of Rome, 00185 Rome, Italy \\ 2 Mathematics Teaching and Education Centre, Faculty of Natural Sciences, Eötvös Loránd University, \\ 1053 Budapest, Hungary; lenart.istvan@staff.elte.hu \\ * Correspondence: alessandro.gambini@uniroma1.it
}

Citation: Gambini, A.; Lénárt, I. Basic Geometric Concepts in the Thinking of In-Service and Pre-Service Mathematics Teachers. Educ. Sci. 2021, 11, 350. https:// doi.org/10.3390/educsci11070350

Academic Editors: Giorgio Bolondi, Luis J. Rodríguez-Muñiz and Federica Ferretti

Received: 6 May 2021

Accepted: 7 July 2021

Published: 14 July 2021

Publisher's Note: MDPI stays neutral with regard to jurisdictional claims in published maps and institutional affiliations.

Copyright: (c) 2021 by the authors. Licensee MDPI, Basel, Switzerland. This article is an open access article distributed under the terms and conditions of the Creative Commons Attribution (CC BY) license (https:/ / creativecommons.org/licenses/by/ $4.0 /)$.

\begin{abstract}
This paper discusses a model of a mathematics teacher professional development implemented in Italy and Hungary with in-service and pre-service mathematics teachers. The model focuses on comparative geometry, and it develops with the use of an artifact: the Lénárt spheres. The teacher training model is the result of several years of experience of the two authors both as regards the activities in the classroom with the Lénárt spheres and as regards the training of teachers in this field. The proposed teachers' professional development, in addition to providing ideas for activities to be implemented in the classroom, has the objective of proposing reflective activities from a community of inquiry perspective; during the activities, mediated by the artifact, both the Pedagogical Content Knowledge and the Mathematical Content Knowledge are taken into consideration (Ball et al., 2008). The model has been implemented in Italy in more than 15 training courses taught in the last 5 years, both with primary school teachers and with secondary school teachers. In Hungary, the model is at the basis of elective courses under the title 'Ball Geometry' at ELTE University, Budapest, for decades. These courses have been aimed at prospective preschool and elementary school teachers at the Faculty of Primary and Preschool Education, as well as future secondary teachers at the Faculty of Natural Sciences. The subject of the teachers' professional development paths corresponds to the comparative geometry between the plane and the sphere. After the presentation of the model, some examples of activities implemented in Hungary during the pandemic period will be illustrated and commented from a didactic point of view, which will serve to exemplify the path described. The described path was carried out remotely in online mode through synchronous and asynchronous activities. The distance obviously changed the way we interacted with the artifact, but it did not prevent the achievement of the courses' objectives.
\end{abstract}

Keywords: Lénárt spheres; comparative geometry; teacher training

\section{Theoretical Background}

As shown in the literature [1,2], the collaboration and mutual trust between teachers and researchers is fundamental for the success of professional development paths, and the design of our training model has taken into account these studies. In all phases of the path, we have tried to create a collaborative and relaxed atmosphere. Our model aims to share specific analytical and reflective practices with teachers with the aim of building a community of inquiry [3,4]. In a community of inquiry, participants are driven by the desire to discover, ask questions, try to understand by collaborating with others in an attempt to produce answers. Everyone, individually or in a group, gives their contribution to the development of the practice through critical reflection activities, and this contributes to the continuous development of the entire community. During the implementation of our training model the teachers and the researchers shared and developed their knowledge; the result of this process is the formation of a shared knowledge of theories and practices composed of elements of mathematics, teaching and pedagogy [5]. In fact, as pointed out by Shulman in 1986, [6], knowledge of the contents of the discipline is certainly central 
to the teaching and learning of mathematics, but, for a teacher, the knowledge of the contents is intertwined with the pedagogical knowledge that is fundamental in the design, development and evaluation of classroom activities. During the teachers' professional development path, all aspects of Mathematics Teachers' Specialized Knowledge [7] are taken into account. In detail, the structure of our model is based on the connection between a group of individuals, that is, the teachers and the mathematics education researcher, and teacher training activities mediated by the artifact Lénárt spheres (see [8,9]).

In this case, we are referring to the meta-didactic transposition model that considers the practices of mathematics educators (researchers) and those of teachers, when both communities are engaged in teacher education activities. It is an adaptation of Chevallard's anthropological theory [10] to teacher education, through the integration of additional elements [11].

The Meta-Didactical Transposition-TMD model is deeply related also with the MKT construct of Ball and others (e.g., [11]).

The complex dynamic interaction develops among various communities involved in teacher education activities, and meta-didactic transposition is characterized by five intertwined features: the institutional aspects, meta-didactic praxeology, the dual dialectics, processes of intermediary processes and the dynamics between internal and external components. All these features allow TMD to consider some of the main teacher professional development paths in relation to their development. One of the cardinal aspects of TMD, which originates within Chevallard's Anthropological Theory of Education is the praxeology. This notion is structured on two levels: Praxis, or knowing how to do, and Logos, or knowledge. Praxis includes the problems to be studied and the techniques to solve them. Logos includes the discourses that describe, explain and justify the techniques used or the production of new ones. Technology is intended in the etymological sense of 'logos on technè', discourse on Technique. The formal justification of Technology is provided by Theory. Every praxeology, therefore, is constructed from the quatern (Problem, Technique, Technology, Theory), with Problem and Technique defining the know-how-Praxis and Technology and Theory defining the knowledge-Logos [12]. Meta-didactic praxeologies do not refer to classroom didactics, but to the practices and reflections on didactic praxeologies that in the various projects are used to train teachers in accordance with a certain theoretical framework and are the result of the interaction between the concrete practices used by teachers, the teachers' own reflection on these practices and the reflection of the community of researchers who are concerned with the effects of the educational processes they develop. And it is precisely the meta-didactic praxeologies that are at the heart of our teachers' professional development courses. Moreover, in this context a fundamental linking figure arises, the broker (mediator), who is one of the elements external to TAD introduced by TMD. The broker is that figure who creates new links between different communities of practice, coordinates them and generates new meanings by facilitating the sharing of knowledge and practices [12]. Trainers in the model we will present below have often taken on the role of brokers.

\section{The Model}

With respect to the design of the activity, the lesson plan we propose during teaching training is inspired by Activity Theory design [13]. The activity plan follows the following schema:

- Introduction of the activity. The researcher discusses with teachers non-Euclidean geometry. Initially, some notions of non-Euclidean geometry are introduced by the researcher from a theoretical point of view (with non-graduate teachers in mathematics we focus more on this point). It is specified that the objective of the training course is not to "learn" non-Euclidean geometry but that we will focus on comparative geometry.

- Manipulative activities with the Lénárt spheres. Teachers are introduced to the Lénárt spheres, and the researcher asks them to begin to familiarize themselves with this structured material. Teachers are divided into groups (usually, a maximum of four 
teachers) and, after an initial exploration moment, the researcher presents some of the features of the sphere that the teachers will use in their inquiry [3]. The researcher addresses the mathematical content selected for the activity from a conceptual and epistemological point of view.

- Analysis of an example. The researcher discusses with the whole group of teachers, according to the rules of a community of inquiry, a didactical path which can be implemented with the use of spheres. Obviously, the paths presented are in line with the school grade in which the teachers teach. The activities presented are analyzed both from the point of view of the content and from the pedagogical point of view, with some of the main mathematical teaching constructs [7].

- Group activity. The researcher assigns a task covering a mathematical content, a learning difficulty and a cognitive process, related to the content at stake, that are developed according to the scholastic level of interest of the teachers. The small group activity is carried out according to the rules of a community of inquiry, and the members of the group strongly interact with the Lénárt spheres. The group activity aims at the construction of a product, an artifact, the design of an activity for students etc., which should highlight teachers' reflections, convictions and beliefs.

- General discussion. The sub-groups present their materials in a written or oral form to the big group. Each presentation is discussed within the community of inquiry, conducted by the researcher, in order to highlight beliefs and convictions, tackle doubts, difficulties and unclear contents regarding both the mathematical content and the analysis of the related learning process from a didactical point of view. The final discussion, based on the oral presentations, is performed with the same characteristics of a community of inquiry.

\section{Venue and Participants}

The present paper is about our experiences with primary and preschool students. We take our examples mostly from distance communication during the last year of the pandemic period. Our main goals have been more or less the same since the course began, but until last March most of the communication with the students happened face-to-face in the classroom. Since the lockdowns of the pandemic, however, we have mainly opted for the written form of communication. The experimentation was conducted with Hungarian and Italian teachers.

With many obvious drawbacks, this method proved surprisingly fruitful in many other ways. Students have time to ponder over an example or to create diagrams or other illustrations much more comfortably than before. Likewise, we could comment on their written answers more comfortably and deeply in this way.

Very importantly, we have tried to introduce a friendly, familiar style that is close to the tone of personal correspondence. We praise good mistakes, by which we mean, in the natural way of truth-finding, perfectly acceptable mistakes through trial and error. In fact, we consider good mistakes to be evidence of independent thinking and experimentation, as opposed to copy-and-paste methods from various internet sources.

The geometry syllabus in the compulsory curriculum fits the future profession of preschool and primary teachers. As such, it contains little or no reference to non-Euclidean geometries that form the basis of the Ball geometry course. One might think that students with this background in math are far from interested in such an advanced subject. This is not the case, as the number of students shows, which was over 80 in recent years.

We regularly ask the participants why they come to the course. The most typical answers are:

- Recommended by someone who has attended before.

- The title "Ball Geometry" made them curious.

- They had a mild or not-so-mild math phobia in secondary school and were hoping for a positive turn in this regard.

- By contrast, they loved math in high school and longed for new challenging subjects. 
Why are these students particularly suitable for checking the level of actual understanding of geometric concepts?

Most of them have adequate wording skills and readability. Consequently, they are able to express their thoughts, feelings, inner struggles with new topics and joy over success.

They are eager for gathering positive mathematical experiences, but at the same time do not feel guilty about deficiencies in their prerequisite mathematical knowledge, since mathematics is just one among many other subjects from pedagogy to literature, art and music in their curriculum. Due to the diversity of their subjects, they were open and receptive to the connection between different areas of human culture and science. Their association skills helped them understand the new concepts.

The shortcomings in their mathematical knowledge turn out to be beneficial in some respects. After appropriate experience, they can recognize their own deficiencies. They rarely escape behind root-learned definitions and arithmetic routines without real understanding. Still, when this is the case, they recognize and correct their own deficiencies. They do not feel the need to be infallible that often causes trouble with future high school teachers. Their answers clearly show what is left in the minds of the educated but not math-specialist students from the mathematical and geometric material they encountered in their previous studies.

It was a great surprise for them to come across a completely different material in geometry than what they were used to. (See Brousseau's idea of the didactic contract.) It was unusual that they could express their opinion on these issues or describe their positive or negative feelings on a mathematical problem. They viewed mathematics and geometry as strictly objective sciences in which only flawless answers can be accepted, and it is up to the instructor to decide whether the answer is flawless.

To be able to judge the effectiveness of the course, we must also consider the following aspect. The basic notions used to introduce students to geometry are indeed some of the most difficult concepts that are among the central problems and heated debates of scientific research in present-day mathematics.

The nature and cardinality of the point, the idea of a continuous line, the definition of a straight line, perpendicular and parallel lines, the concept and measurement of distance and angle-all of them are light years away from being simple or easy.

Yet, school education expects the learner to accept these terms as obvious concepts without any doubt or objection.

This can be one of the first experiences that alienate the student from geometry. He gets used to keeping his doubts and objections to himself. Since the definitions given by the teacher do not satisfy him, he does not recognize the geometric shape by the definition, but only by the visual image. He views the definition as a meaningless but necessary formality, a game to play because the teacher requires him to do so.

The efficiency or ineffectiveness of visual recognition is revealed when the student encounters the same concept in a different environment, by a different form of representation. The visual appearance that helped the student recognize the concept becomes one of the main obstacles in the new environment to true understanding, to the process of successful interiorization.

However, if the student overcomes this obstacle and identifies the same concept in the new environment, the failure turns into a success. He understands the significance of the definition and breaks free from the shackles of inoperative knowledge within a single system.

If current math teaching considers it its main task to make students know certain fixed concepts and theorems, then comparative geometry is a new subject inside or outside of mathematics. Its purpose is not to convey an irrefutable truth established by the great scholars of the past, but to inspire the student to think independently, to express his or her opinion, to make decisions, to be able to describe his or her own line of thought, to make mistakes without a sense of guilt, but with the intention of correcting the mistakes. 
Summing it up, comparative geometry views the student not merely as a pupil of great scientists of the past but as their partner. It is not because the student considers himself as smart as they (who knows, anyway? they were once pupils too), but because those great scientists lived and acted the same way, considering their predecessors not as their superiors but as fellow researchers in their investigations.

\section{Some Extracts from the Experimentation}

We report below some of the discussions with teachers regarding some topics of geometry, in the perspective of the comparison between plane geometry and spherical geometry.

\subsection{What Is a Point?}

Student 1: "In my opinion, a point can be a physically drawn point on a flat surface, such as a drawing sheet. But it can also be a reference point, for example, I choose the chandelier on the ceiling as a reference point for how far I am from it. If I wanted to be very philosophical, I would say that the point can be anything, and the nothing, the emptiness is everything. I don't know, this came to my mind first. I'm a little shot at the question of drawing half a point ... Except for the 'half point' drawn with a pen, you might not be able to draw half a point because it's already a point."

Teacher: "Nice, illustrative description. I agree with you that half a point cannot be drawn. I once thought that I was the first to tell this, but I was a little mistaken: a Chinese book Mo Jing already had it 2300 years ago..."

Student 2: "I would choose the circle as my best friend to build geometry on the plane. It is easy to create triangles from it, all kinds of rectangles or even polygons by drawing different radii, diameters and/or secants... Well, if the point was the 'good' solution, then I am very far from the truth..."

Teacher: "Dear M., one of my main goals in this course is to convince you that the choice of basic concepts is completely arbitrary!"

Student 2: "I think the point is the smallest possible shape, the point is a point on a flat surface and on a spherical surface. Each additional shape consists of points and is made up of points, starting with a first point and ending with a last point. What is the difference between point and nothing, it is a catchy question? The point is something, it exists and is there, it is a place, so by no means nothing."

Teacher: "I don't know much more myself!"

Student 3: "If we consider an infinite flat surface even the point is infinite itself. I think a point that could be visible on a surface is itself full of points. Probably 'half of a point' it's not a naked-eye measurable element, neither an abstract idea we can think about. I mean I consider they are both 'infinite' surfaces (with no boundaries) and we draw a point this point is, in an abstract point of view, infinitive full of points. As we can see, it isn't possible to consider 'half of a point', a point it's always full of infinite little points itself. I can conclude a point is a 'relative idea of element', it depends on the position we want to consider it."

Teacher: "My answer (which you can accept or refuse) is that there is only one thing that I know for sure about the point, namely, its location within a larger environment. The dot or cross that symbolizes a point on a sheet of paper or on the peel of an orange is actually a sea of ink or paint when examined under a magnifying glass or a microscope. It is up to the user to decide whether or not a particular shape can be considered a point."

Student 4: "The simplest element on the plane has to be two-dimensional and, on the sphere, three-dimensional. First, I wanted to choose a square because in my head I compare a flat surface always with a sheet of paper but then I thought about it again. I think I would choose a circle because it has no beginning 
and no end just like the infinite surfaces. Three-dimensional it would become a bullet then."

Teacher: "In this course we take the point as the simplest element on the plane and on the sphere. What is a point? What is the difference between the point and nothing?"

Student 4: "A point is a point, no nothing. How could it? Points can be very important. For example, in a text they mark the end of a sentence or are part of a sign (!) or letter (i). You use them also for writing the date (26 February 2021). The display of a computer/laptop/tablet/mobile phone consists also of kind of 'dots' (pixel). If you look at one of these 'dots' alone it doesn't seem important but on the whole, they form a picture. Maybe every point consists out of many other points. In that case a half point is just a point out of less other points."

Students are surprised, sometimes shocked, by the question. They think that the concept of point needs no further explanation. Many math teachers are likely to share the same view and will not initiate a discussion with students on this issue. In our opinion, an open exchange of ideas between teacher and student has several benefits. As highlighted by the TDA [4], both in terms of Praxis and Logos, the role of the teacher is very relevant, both in terms of the choice of knowledge involved and the approaches to it. The teacher cannot and should not play the role of infallible authority, but only represent his own perception, his approach to definition, which in no way excludes other opinions. An exchange of views at the beginning of the geometry class creates a student-friendly atmosphere in the classroom, promotes the independent expression of different opinions and encourages students to exercise the right to trial and error during their investigations.

Teacher's attempt to interpret the notion of point to all students:

"If I say: 'New York is an important point of railway junction within the U.S.A.,' this means that nothing else is interesting for me of New York (its history, population, rivers, etc.), but only its place within the U.S.A. In other words, I consider New York as a point within the U.S.A.

If I say: 'Time Square is an important point of traffic within New York,' I consider Time Square as a point within New York. In this case, Time Square relates to New York in the same way as New York to the U.S.A. in the previous example.

When I say, 'The Earth orbits the Sun in an elliptical orbit,' I am viewing the Earth as a point because I am only focusing on the location of the Earth in the Universe. However, when I try to explain why there are seasons or days and nights on the Earth, the point assumption helps me no longer. I have to assume the movement and rotation of a threedimensional sphere as the shape of the Earth."

\subsection{What Is a Straight Line on the Plane and on the Sphere?}

Teacher: "In order to study the straight line, connect two points with the line which shows the shortest distance on the surface. Stretch a rubber ring on a flat surface (cell phone) and on a sphere (an orange). Also, follow the route of a drop of water on a flat surface and on a sphere. Describe the lines you get."

Student 5: "If we draw a line on a drawing sheet, i.e., we connect 2 points we get a line. I think you can extend both ends of the point indefinitely. I couldn't tell a given distance how long, actually, as long as we can, we'll pull the extension. Since we are working on a flat surface, the lines do not meet each other, but as we live on the Earth, which is spherical, so if I draw a straight line on Earth and continue, I may come back to one end once... If we draw a line on the spherical surface, we get a circle from above, for example, and I don't think that line can be extended because they meet on the sphere (the starting point will be the end point.) These lines are different on the planar and spherical surfaces but are still the same. In the same way, we draw a line, they will only have a different look/shape. On a flat surface, it only looks like a straight line, and on a spherical 
surface, it looks like a circle from above. And on a plane, nothing resembles a circle, I just see the drawn lines as straight."

Student 6: "The simplest line on the plane and on the sphere is again the point. If we consider the point as the simplest element on the surface, the succession of points is a line, if we go 'dividing and deconstructing' the line into consecution of points one point is in itself a line. So, the simplest line is the point. I don't know if you'll agree but I think that we can't consider the straight line as the infinite flat surface. I mean... A line, a consequence of points it's something, it can't be considered as all the flat surfaces. Honestly, it's a difficult dissertation even with myself! Seen in depth a line is a line (consequence of points) either on the plane or on the sphere."

Teacher: "This 'consequence of points' is very hard to define... Is there a bisecting point between any two 'consecutive' points? If yes, what is the meaning of 'consequence'?"

Student 6: "I wrote it basically starting from the point I wrote before, yes I think ideally we must consider a bisecting line between two points. I wrote the answer considering the assumption I did before: 'the line is a consecution of points, so one point is in itself a line. So, the simplest line is the point'."

Student 7: "What is the next simplest element on the plane? I first, again, wanted to choose the square. I think because it's one of the shapes you always hear of in school. But then I read my answer from the question above again and realized that a square could consist out of many points. Just like on the computer desktop. So, I am not sure about my answer because now I think that every other element could consist out of points."

Teacher: "What is the simplest line on the plane and on the sphere?"

Student 7: "I think the simplest lines are these consisting out of points."

Teacher: "But all lines consist of points! Which is the simplest among them? I proposed the straight line on the plane and asked if there is a straight line on the sphere. What do these lines look like on the plane and on the sphere?"

Student 7: "On the plane the line is totally straight no matter of which direction you look at it. On the sphere the line is only totally straight if you look at it from a $90^{\circ}$ point of view which is directly above the line. Otherwise, you can see 'roundings'."

Teacher: "Again, you cannot look at it from outside, you are a 'spherelander' living on the sphere."

Student 8: "Every line is different, because in every situation the line behaves in different ways. It is easier to draw a straight line on a flat surface. Besides, it is much nicer on the plane than on the sphere. It was also clear from the last task (orange drawing) that it is more difficult to draw a straight line on a spherical surface. The lines are similar in plane and sphere, but a spherical line is not a regular line, but takes on the shape of the surface."

Teacher: "Whether it is more difficult or whether it is nicer is probably a matter of habit. My Vietnamese acquaintances did not understand why I cannot eat with chopsticks because it is so simple..."

This is also a topic that many teachers do not waste much time explaining. They probably think the visual image is enough to decide if the line is straight. This is in stark contrast to one of the central tasks of math lessons, namely to clarify the meaning of the mathematical definition, which remains a lifelong mystery to many children and adults. The transfer of the concept of straight line from the plane to the sphere offers the teacher an excellent opportunity to convey the essence and advantages of mathematical definition to the students. 
Another advantage is that there are several experiments with strings, rubber rings, drops of water, etc. to illustrate a straight line on the plane and on the sphere. In this way, the teacher has the opportunity to emphasize the role of direct perception and experience in mathematics, the path from concrete experiment to mathematical-geometrical abstraction. In this situation, the teacher's fundamental role as a broker emerges [10].

\subsection{When Do You Call Two Straight Lines Parallel?}

Student 8: "On a plane surface, two lines are parallel if the line lies at the same angle below or above the specified line. On a spherical surface, it could only be parallel if we spread the surface and compared the lines drawn below or above each other there." "Very clever, very deep thought. Parallel means that if we connect two straight lines the same points will be equidistant from each other."

Student 9: "When are two planar lines parallel to each other? When the points of the two lines at the same height are equidistant from each other, they do not meet each other by dragging them to infinity, that is, they have no point of intersection. When are two spherical lines parallel to each other? When the two circular arcs have no intersection with each other and all points closest to each other are equidistant from each other, they are the same size. What is the meaning of 'parallel' anyway? It is the location of the nearest points of two adjacent lines at equal distances, without any point of intersection."

Student 10: "Two plane lines are parallel if they do not intersect and are monoplanar. Unfortunately, for the sphere, I don't know if there is any example of a parallel line at all."

Student 11: "Two lines on the plane are parallel if there is the same distance between them throughout in a plane: there is the same distance between two lines all the way, not necessarily the same length. On the sphere it is just as in the plane, the Tropic of Cancer is parallel to the Tropic of Capricorn, but so are the Equator and the polar circles."

Student 12: "Which straight lines are called parallel on the plane or on the sphere? Two straight lines are parallel that never touch themselves. Actually, I never heard about parallel lines on the sphere. All the straight lines on the sphere have the same path/direction."

Student 13: "We consider two lines or any other things to be parallel that are not in contact with each other, but if we put them on top of each other, they would exactly overlap."

Teacher: "Are the edges of two plates on the table parallel to each other?"

Student 13: "Two planar lines can easily be like this, but we cannot do this on a spherical surface. "

Student 14: "Two plane lines are parallel to each other if there exists a line that is perpendicular to both of them."

Teacher: "Excellent definition (although other types are also possible)."

Student 14: "Two spherical lines are parallel to each other if there exists a line that is perpendicular to both of them."

Teacher: "I am in trouble now what to answer! According to your definition, which spherical straight lines are NOT parallel to each other? You see, it is up to us what we call parallelism..."

The concept of parallel lines also seems perfectly clear to the student and often to the teacher as well. The two lines do not intersect and keep an equal distance from each other-that needs no further explanation. However, after the discovery of hyperbolic geometry, the general public has met explanations about parallelism that were incomprehensible 
for most readers, including many students and teachers. The concept of the spherical straight line gives the teacher a tool to examine the question of parallels from a different perspective. Although spherical geometry differs from plane geometry and hyperbolic geometry in terms of parallel lines, stepping out of the box of Euclidean geometry helps a lot in understanding the fundamentals of a third world of geometry. In terms of TDA, the praxeologies inherent in non-Euclidean geometry can only consolidate knowledge of Euclidean geometry and, thus, lead toward a more enlightened learning.

\subsection{How Many Full Straight Lines Can Be Drawn Through Two Points on the Plane and on the Sphere?}

Student 15: "How many full straight lines can be drawn through two different plane points? Only one.

How many full spherical straight lines can be drawn through two different spherical points? Two full lines can be drawn. From one point to another on the shortest path and the other line from the other half of the point (as if continuing the shorter line) is connected to the other point."

Student 16: "I think an infinite number of straight lines can be drawn, especially on a flat surface."

Student 17: "If we draw two points on a sheet and try to draw lines on the paper, we can connect the two points with a specific line from point to point. If we do the same on a spherical surface and two points are selected in the same way, then (1). draw a line between two points that can be approached from two sides, that is, we can choose from two lines if the two lines are not of equal length (2). If the two lines are of equal length (two points are equidistant), then it does not matter which line we choose, we are talking about the same line in both cases."

Student 18: "On the plane: just one full straight line. On the sphere: if we consider the points which stand on the 'great circle' (or the shortest path between two spherical points on the sphere) there are infinite spherical straight lines which passes through them. Since we said that the only line on the sphere that can be considered as a 'spherical straight line' is the one which passes through Great circle, this is the only possibility we have. "

Teacher: "I am a bit confused by your wording, so I reformulate the question: How many full spherical straight lines = great circles can be drawn on the globe through Bologna and Moscow?"

Student 18: "None, they're not on the Equator so the line which passes thorough Bologna and Moscow is spherical circles not great line."

Teacher: “No, No, No! Now I see your problem: it is your reminiscences from geography about the latitude lines. If the Earth-globe is an orange, and you pick two toothpicks into Bologna and Moscow, of course there is a spherical great circle through them! In the geographic coordinate system, the spherical straight lines are the Equator and all longitudes (meridians). Nothing else-no Tropic of Cancer or Capricorn, no Arctic or Antarctic Circle. Of course, there are infinitely many other great circles which are neither north-south (like the longitudes), nor east-west (like the Equator). If you understand this, then you can go through all the following questions to correct them. Do not rush, take your time! And: Is this the only possibility, or can we find special pairs of spherical points with different answer to the same question?"

Student 18: "If we consider two points on the Equator (for ex Quito and Padang) then we can say the line which passes through them is a spherical straight line. In this case one straight line can be drawn."

Teacher: “See my ‘No, No, No!' remark above!” 
This question establishes the link between the description of the point and the straight line. When the teacher has discussed the concept of the point on the plane, the student will likely think that the point behaves the same way on the plane and on the sphere. This seems to be confirmed by the fact that two points on the sphere usually define a straight line, as is always the case in the plane. The discovery of the opposite points and the infinite number of lines passing through them is extremely impressive for the students. Stepping out of the plane onto the sphere, the teacher has a way to show the student that even the simplest geometric shapes, such as the point, can show sharp differences in different geometries. The discovery of the opposing points and the infinite number of straight lines that connect them is extremely impressive for the students. By stepping out of the plane onto the sphere, the teacher demonstrates that even the simplest geometric shape behaves quite differently in different geometries.

\subsection{Is There a Smallest Circle on Plane and Sphere?}

Student 19: "I don't think that the smallest circle exists on the plane. What circle can be considered as the smallest? We can always draw a circle which is smaller than the little one, then an even smaller circle. On the sphere there is no smallest circle either, because all the circles which are different from the great circle are all small circles."

Student 20: "I don't think there can be an infinitely large or an infinitely small circle on the plane. On the sphere I think there is such a circle, the latitudes and the polar circles came to my mind."

Teacher: "The polar circles are indeed much smaller than the Equator, but are there even smaller spherical circles (even if they don't have a special geographic name)?"

Student 21: "The smallest circle on the plane is a circle one point away from the point (the radius of the circle is a point)."

Teacher: "Cute wording! Still, the point is often called a degenerate circle because no matter how small a circle we draw, it has an even smaller circle, all the way to the center of the circles. In other words, we can approach the point as close as we want with real circles. In general, this approach can also be considered as the definition of the adjective 'degenerate'."

Student 21: "Likewise, on the sphere we draw a circle of radius one point away around the point."

Student 22: "I would say the smallest circle on the plane is a circle which only consists of four points which are connected through straight lines."

Teacher: "Lovely childish! (Sorry, I could not resist, do not be offended!) Can four points form a full circle? And if you take the bisection of the sides, do you get an even smaller 'circle'? But your answer is a wonderful example of how deeply Euclid's is ingrained in our thinking. You believe in the Euclidean straight line, even if you define a circle! Now on a serious note: Is there a real circle on the plane so you can't draw another circle entirely within the original circle? And so on and so on? What is the end of this process?"

Student 23: "Surely there is, the smallest circle I can represent on the sheet. It could be an empty dot."

Teacher: "In this geometry game, we are dealing with things that only exist in our imagination! The point is not an empty circle, as there are still an infinite number of points inside the empty circle. We can call the point the smallest circle or a degenerate circle, because no matter how small a circle is, we can draw or imagine an even smaller circle, all the way to the center of the circles." 
This, too, seems to be a topic that needs no explanation, although the concept of limit is one of the guiding themes of recent mathematical research. The vast majority of students are just as unsure of this question as mathematicians and philosophers have been for thousands of years. Students often claim that the smallest circle depends on how sharp the pencil we draw the circle with is. It is also a common answer that the radius of the smallest circle is 1 unit of measurement. Both the plane and the sphere give the teacher an opportunity to start a discussion with his students about infinitely small geometric shapes and infinitely small numbers, i.e., the concept of geometrical and arithmetical limit. Interestingly, the role of $\pi$ as the circumference/diameter ratio is characteristically different on the plane and the sphere. So, the role of pi can be examined by the teacher by referring not only to abstract arithmetic considerations about rational and irrational numbers, but also to direct geometric experimentation and measurement.

\section{Discussion and Conclusions}

As emphasized by the TDA, in teaching situations it is good to keep several factors under control. The Technology and Theory that define the Know-How-Logos are as important as the Problem and Technique that define the Know-How-Praxis [10]. The teachers' choice of the knowledge to be transposed is crucial. As the above excerpts show, there is a lack, as far as the students involved are concerned, of both a conscious knowledge of geometry and a habit of collective discussion and reflection during the learning paths.

In full accordance with Wagner and Jaworski, as cited in Jaworski [3], we emphasize the importance of co-learning (as was called in the above-mentioned paper) between the educator and the practitioner, somewhat similar to the relationship between a theoretical scientist and an experimental physicist, which can, of course, be realized within one and the same person. This relationship should be based on the cooperation of equal partners, instead of a hierarchical subordination between the scholar and the teacher. The hierarchical relationship can be harmful in several ways. It may give a sense of inferiority and irritability to the practicing teacher, who may come to feel that his direct experience in day-to-day labor in the classroom becomes an impersonal statistic in a scientific dissertation. His role is reduced from that of a research fellow to that of a research object, regardless of his own inventiveness and creativity and his mathematical and pedagogical skills. Another danger is that the practicing teacher perceives the educator's behavior as a model for his own attitude, and creates a similarly rigid, hierarchical atmosphere in the classroom. He confines himself to infallible statements instead of experimenting, reasoning and arguing, and expects unquestioned acceptance from his students. This can be all the more inconvenient and alienating for the student, since the concepts studied in school geometry are often among the most difficult ones, and have been the focus of intense scholarly debate to this day. In the examples above, we have seen how much uncertainty and doubt arise in the students about seemingly obvious basic concepts of geometry, such as the point, the straight line, the circle, etc. If we do not allow these doubts to come to surface and be clarified, the student is likely to feel all further conclusions and theorems to be uncertain and unconvincing. The changed relationship between teacher and student should also be reflected in the oral or written communication between them. We are by no means advocating a disrespectful manner, that may be tolerable among peers but unacceptable between teacher and student. We advise to teachers to communicate in the style of live language and, as far as only possible, avoid using outdated terms that are not present in everyday language.

As TDM suggests and as outlined in the excerpts above, all this is transposed and amplified when in a teacher education context. The role of the teacher educator as a broker becomes essential when there is a shift from sapient knowledge to the mathematical and pedagogical knowledge necessary for teaching, i.e., when the object of the transposition becomes the praxeologies related to the teaching-learning of mathematics.

Author Contributions: Writing—original draft, A.G. and I.L.; Writing—review \& editing, A.G. and I.L. All authors have read and agreed to the published version of the manuscript. 
Funding: This research received no external funding.

Institutional Review Board Statement: Not applicable.

Informed Consent Statement: Informed consent was obtained from all subjects involved in the study.

Conflicts of Interest: The authors declare no conflict of interest.

\section{References}

1. Jaworski, B.; Huang, U.R. Teachers and didacticians: Key stakeholders in the processes of developing mathematics teaching. ZDM 2014, 46, 173-188. [CrossRef]

2. Krainer, K. Teachers as stakeholders in mathematics education research. In Proceedings of the 35th Conference of the International Group for the Psychology of Mathematics Education, Ankara, Turkey, 10-15 July 2011; pp. 47-62.

3. Jaworski, B. Research practice into/influencing mathematics teaching and learning development: Towards a theoretical framework based on co-learning partnerships. Educ. Stud. Math. 2003, 54, 249-282. [CrossRef]

4. Wells, G. Dialogic Inquiry: Towards a Socio-Cultural Practice and Theory of Education; Cambridge University Press: Cambridge, MA, USA, 1999

5. Jaworski, B. Development of the mathematics teacher educator and its relation to teaching development. In International Handbook of Mathematics Teacher Education; Jaworski, B., Wood, T., Eds.; Sense Publishers: Rotterdam, The Netherlands, 2008 ; pp. $335-361$.

6. Shulman, L.S. Those who understand: Knowledge growth in teaching. Educ. Res. 1986, 15, 4-14. [CrossRef]

7. Ball, D.L.; Thames, M.H.; Phelps, G. Content knowledge for teaching: What makes it special? J. Teach. Educ. 2008, 59, 389-407. [CrossRef]

8. Lénárt, I. Comparative Geometry in distance education. J. Phys. Conf. Ser. 2021, 1840, 012003. [CrossRef]

9. Rybak, A.; Lénárt, I. Hungarian perspectives. Comparative Geometry in Primary and Secondary School. In The Pedagogy of Mathematics; Webb, P., Roberts, N., Eds.; MISTRA and Real African Publishers: Johannesburg, South Africa, 2017; pp. 107-124.

10. Chevallard, Y. Transposition Didactique du Savoir Savant au Savoir Enseigné; La Pensée Sauvage Éditions: Grenoble, France, 1985.

11. Aldon, G.; Arzarello, F.; Cusi, A.; Garuti, R.; Martignone, F.; Robutti, O.; Sabena, C.; Soury Lavergne, S. The Meta-didactical transposition: A model for analysing teacher education programs. In Proceedings of the PME37 Conference, Kiel, Germany, 28 July-2 August 2013; Volume 1, pp. 97-124.

12. Arzarello, F.; Robutti, O.; Sabena, C.; Cusi, A.; Garuti, R.; Malara, N.; Martignone, F. Meta-didactical transposition: A theoretical model for teacher education programmes. In The Mathematics Teacher in the Digital Era; Clark-Wilson, A., Robutti, O., Sinclair, N., Eds.; Springer: Dordrecht, Germany, 2014; pp. 347-372.

13. Radford, L. Methodological aspects of the theory of objectification. Perspect. Educ. Matemática 2015, 8, 547-567. 